EUROPEAN JOURNAL OF PURE AND APPLIED MATHEMATICS

Vol. 13, No. 1, 2020, 69-83

ISSN 1307-5543 - www.ejpam.com

Published by New York Business Global

\title{
Finite Element Simulation of Newtonian and Non-Newtonian Fluid through the Parallel Plates Affixed With Single Screen
}

\author{
Abid Ali Memon ${ }^{1}$, Muhammad Asif Memon ${ }^{1, *}$, Kaleemullah Bhatti ${ }^{1}$, \\ Gul Mohammad Shaikh ${ }^{1}$ \\ ${ }^{1}$ Department of Mathematics, Sukkur IBA University, Pakistan
}

\begin{abstract}
In the contemporary research article we have performed a numerical investigation of the non-Newtonian fluid flow through a rectangular channel with a fixed solid screen devoted at the angles 100 to 450 degrees. We have employed the power-law model for shear thickening and shear thinning fluids with the high Reynolds number between 1000 and 10,000. The obstacle has been solved by putting in the Galerkins least square strategy of the finite element method and the procedure has been carried out utilizing the commercial software COMSOL Multiphysics. Various flow properties such as 'maximum flow rate' and 'pressure' have been discussed in the terms of the Reynolds number and also using the linear and quadratic regressions in order to establish the relationship between them for the future analysis. Moreover the impact of turning screen in the shape of increment in the maximum flow rate and pressure is checked in terms of Reynolds number and Satisfactory results are gained in comparison with the results available in the literature.
\end{abstract}

2020 Mathematics Subject Classifications: 76A02, 76A05, 76M10

Key Words and Phrases: Finite Element Analysis, Newtonian Fluid, Non-Newtoninan Fluid, Parallel Plates, Embedded Screen

\section{Introduction}

The screens are routinely used in the propellant tanks as filter devices in the ground of spacecraft and aerospace engineering. Although as the fluid enters into any media or region, it is usually problematic to monitor and switch over the flow rate and pressure exercised in the region. For the motive, the solid screens or perforated plates have the dominant role in the concern. The screens are the outfits which comprise the pore in it, having the tendency to overturn the tangential component of the velocity field to escalation the speed of the fluid possess the resistance and refraction coefficient as the characteristic

*Corresponding author.

DOI: https://doi.org/10.29020/nybg.ejpam.v13i1.3586

Email addresses: asif-memon@iba-suk.edu.pk (M. A. Memon), abid.ali@iba-suk.edu.pk (A. A. Memon) 
parameter in it. It is also recognized that the viscosity of the fluid and the angle of inclination at which the screen is placed in the media play an extensive role to optimize the velocity and alleviating pressure as well. Due to these applications, many researchers and mechanical engineering scientists put their endeavors to apply various kind of mathematical methodology or carry out a lab experiments to make it possible to check the behavior of fluid while passing through the screens. At this place, we are attending to present some literature review.

By the execution of an experiment for scrutinizing the fluid flow through the arbitrarily shaped screen named 'gauze wire', Elder [2] found an asymptotic solution for the small-angle that uses numerically calculated velocity, mean inlet velocity, the angle at which the screen attached, resistance coefficient and refraction coefficient. Dissimilar fluids like air, nitrogen and water, the Dutch Twilled Screens (DTS) along with the Broad Mesh Geometry were investigated to forge the association between the Euler number and Reynolds number by A. Fischer and Gerstmann [3]. Practicing the Discrete Element Method (DEM), Sawant et al [4] investigate the fluid behavior through the screens broken down to the screens settled at the specific angles when the rough particles are in contact with them. He obtained the capability of mass transfer getting better when they are observed through the improvement of the angles of the screens. Consideration is given by Santiago and Wang [1], they compare the relationship between the head-loss in terms of the angle when the fluid flow was analyzed. They discover that both elements own a direct relationship with each other i.e. when one thing is improved other to get the enhancement. A lab experiment was carried out by the Teitel [7] to assay the fluid flow through the screen when it is attached at the angle from 450 to 1500 settled at the middle of the channel using the porosity (range: $0.4-0.62$ ). The experiment was also confirmed through the ANSYS CFX-II, found that the mass flow rates for each porosity and concluded that the mass flow rate at the angles 45 degrees and 135 degrees is same. A new and general formulation to solve the compressible and in-compressible flows through an integrated approach was recommended by Huake and Haughs [5], recognized as Galerkins Least Square (GLS) scheme. Rendering to it if velocity, pressure, and temperature are the primitives variable then a standard formulation can be done with the custom of finite element method along with the least square scheme. Abid, et al [6] with the implementation of Galerkins scheme using the package of commercial software Comsol multiphysics 5.4 analyzed the Newtonian and in-compressible fluid flow through the rectangular channel containing three screens. They spotted that by adding more screen in the channel the flow can speed up and at the end, they cast a light on the drag force exerted by the three screens and produced the empirical relation with the angles.

The intention of the existing study is to canvas the fluid flow through two fixed parallel plates imposed with screen boundary conditions. The problem is steady-state, incompressible and laminar. We have implemented the Power-Law model to testify the shear thinning and shear thickening flows. The case of Newtonian fluid flow under the same conditions has also been investigated similarly. Fluid properties like the maximum 
flow rate and maximum pressure are discussed in comparison to increasing Reynolds number ranging from 1000 to 10,000 keeping the resistance and refraction coefficients of 2.8 and 0.78 respectively.

\section{Methodology}

\subsection{Geometrical Construction and Meshing.}

A block at the middle of parallel plates is laid which is mobile from 100 to 450 degrees and the medium has the aspect ratio of $4: 1$ between lengths to widths. The upper and lower boundaries are the walls and slip boundary conditions are imposed. The right wall of the channel is made out as the inlet boundary condition from where the average inlet velocity is allowed to enter depending on the Reynolds number, while the left wall is facing outlet boundary conditions with zero pressure considerations. The geometrical structure is shown in Fig. 1(a).

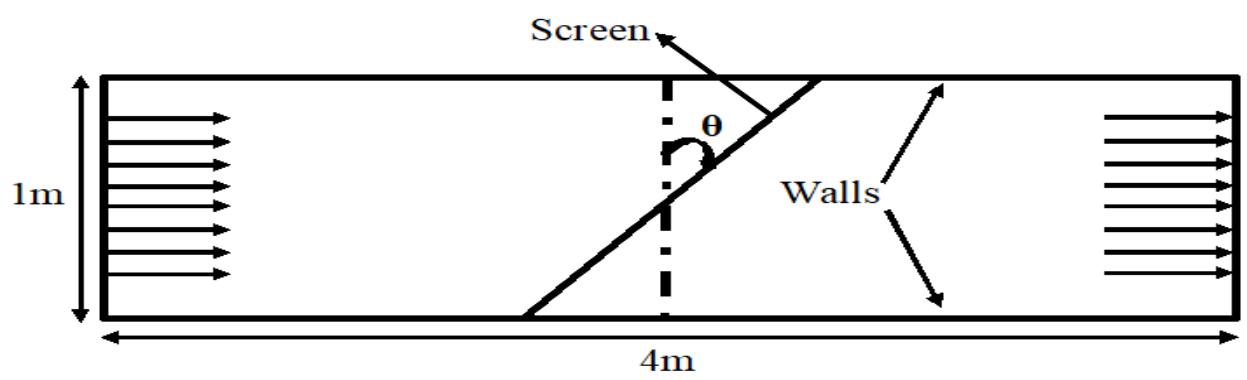

Figure 1: The Geometrical structure of the investigated channel

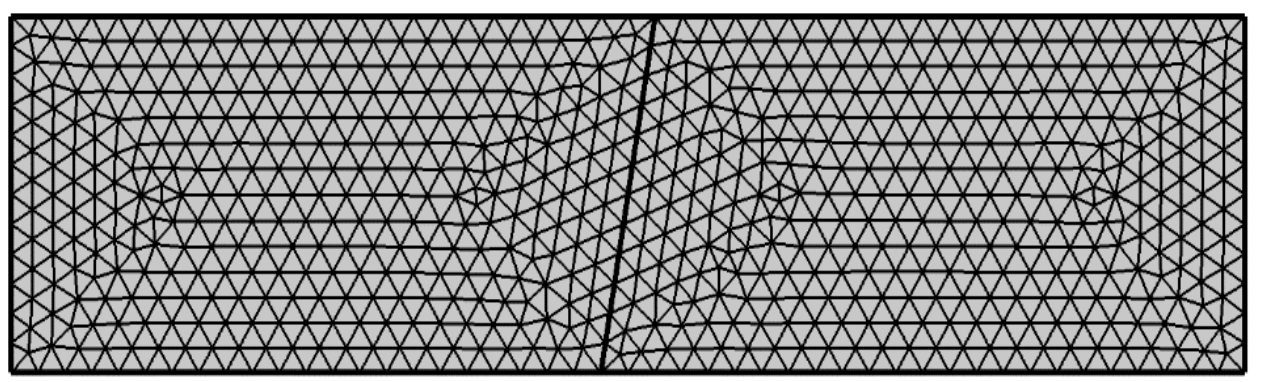

Figure 2: Meshing Structure of Geometry with Irregular Triangular Elements

The further step is of meshing the designated geometry with convenient elements to get the numerical resemblances where their linear shape function. Due to the absence of complications of the geometry and acquiring more specific and minimum error results, the irregular triangular elements are adopted to divide a large domain. The mesh structure of the geometry is shown in Fig. 1(b) and the statistics are listed in Table 1. 
Table 1: Meshing Description Table

\begin{tabular}{|l|l|}
\hline \multicolumn{2}{|c|}{ Meshing Description } \\
\hline Property name & Value \\
\hline Minimum quality element & 0.7629 \\
Maximum quality element & 0.9793 \\
Number of triangular elements & 2382 \\
Edge elements & 215 \\
Vertex elements & 10 \\
\hline
\end{tabular}

\subsection{Governing equations and Regression Equations.}

In order to monitor the two-dimensional flow through the screen when affixed at the middle of the parallel plates, the in-compressible Navier Stokes equations are discretized by practicing the pragmatic and nascent method of finite element advent known as Least Square Galerkins scheme. It has been decades; the Navier Stokes equation is assisting to analyze the behavior of fluid flow problems by satisfying the different boundary conditions. Expected to the non-linearity because of convective terms in it including a second-order partial derivatives, the Navier Stokes equations are too challenging to get an exact solution without thinking of some simplifying assumption which happen rarely in practical demands. Numerical methods contribute most in this regard.

In the current problem, the simulation and modeling are carried out through the COMSOL Multiphysics. The problem is steady-state taking the continuity equation and momentum equations which are set out as:

$$
\begin{gathered}
\frac{\partial \vec{V}}{\partial t}+(\vec{V} \cdot \nabla) \vec{V}=-\frac{1}{\rho} \nabla P+\mu \nabla^{2} \vec{V}+F, \\
\nabla \cdot \vec{V}=0 \\
\frac{\partial \vec{V}}{\partial t}=0 .
\end{gathered}
$$

Where equation (1) is known as momentum equation and equation (2) is known as continuity equation. In these equations $\vec{V}=\langle u, v\rangle$ is the fluid velocity with $u$ and $v$ as horizontal and vertical component respectively. Here $\mu$ and $\rho$ are the viscosity and density of the air respectively. The non-Newtonian fluid is tested using the power law model in which the viscosity is described as:

$$
\begin{aligned}
& \mu=m(\lambda)^{n-1}, \\
& R e=\frac{\rho u_{i n} L}{\mu} .
\end{aligned}
$$


Equation (4) will be implemented in equation (5) and then we have a strong formulation for non-Newtonian model. The results will be validated by using the asymptotic solution developed in [2]. Slip boundary conditions will be implemented in order to avoid the viscous effects near these boundary. Hence by considering the normal vector $\vec{n}$ to the velocity field we can define the slip boundary conditions as follows:

$$
\begin{gathered}
\vec{V} \cdot \vec{n}=0, \\
\vec{K}-(\vec{K} \cdot \vec{n}) \cdot \vec{n}=0,
\end{gathered}
$$

where

$$
\vec{K}=v\left(\nabla \vec{V}+(\nabla \vec{V})^{T}\right) \cdot \vec{n} .
$$

It has been decades that the screens are used to control the fluid speed through the medium. Screens are the components which have tendency to suppress the tangential component of the velocity which results into little boost of the speed of the fluid. The screen boundary conditions are described by the following equations:

$$
\begin{gathered}
{[\rho \vec{V} \cdot \vec{n}]_{-}^{+}=0,} \\
\left.\left[\rho-(\vec{n})^{T} K \vec{n}+\rho(\vec{V} \times \vec{n})^{2}\right)\right]_{-}^{+}=-\frac{k}{2} \rho-(\vec{V}-\times \vec{n})^{2}, \\
\vec{n} \times \vec{V}+=\eta\left(\vec{n} \times \vec{V}_{-}\right) .
\end{gathered}
$$

In these equations " + " and " - " show the presents of the parameter up and downstream respectively. The impacts are tested using the non-dimensional Reynolds number and methodology is applied to solve these governing equations subject the boundary conditions as given in [5].

After implementing the Galerkins least square scheme on the constitutive equations using COMSOL Multiphysics, the results are analyzed by regression procedures. Here we will find the empirical equations that we can determine for the maximum flow rate and for the maximum pressure in terms of the Reynolds number called regression lines by applying the linear regression and quadratic regression procedure. The procedures are as follows:

Let $R_{e}=x$ and $V_{\max }=y$ or $P_{\max }=y$ then we can define the average as follow:

$$
\text { mean: } \bar{x}=\frac{\sum x_{i}}{n}, \bar{y}=\frac{\sum y_{i}}{n} \text { and } \overline{x^{2}}=\frac{\sum x_{i}^{2}}{n} .
$$

If we define the following variations:

$$
\begin{gathered}
S_{x x}=\sum x_{i}^{2}-n \overline{x^{2}}, \\
S_{x y}=\sum x_{i} y_{i}-n \bar{x} \bar{y},
\end{gathered}
$$




$$
\begin{aligned}
S_{x x^{2}} & =\sum x_{i}^{3}-n \bar{x} \overline{x^{2}}, \\
S_{x^{2} x^{2}} & =\sum x_{i}^{4}-n \overline{x^{2}} \overline{x^{2}} .
\end{aligned}
$$

Then we can define the best quadratic fit by the equations:

$$
y=A+B x+C x^{2} .
$$

Where the coefficients $\mathrm{A}, \mathrm{B}$ and $\mathrm{C}$ are calculated using the following relations:

$$
\begin{gathered}
B=\frac{S_{x y} S_{x^{2} x^{2}}-S_{x^{2} y} S_{x x^{2}}}{S_{x x} S_{x^{2} x^{2}}-\left(S_{x x^{2}}\right)^{2}}, \\
C=\frac{S_{x^{2} y} S_{x x}-S_{x y} S_{x^{2} x}}{S_{x x} S_{x^{2} x^{2}}-\left(S_{x x^{2}}\right)^{2}}, \\
A=\bar{y}-B \bar{x}-C \overline{x^{2}} .
\end{gathered}
$$

For the linear regression to fit the line:

$$
y=a+b x .
$$

Where $a$ and $b$ are calculated using the following relations:

$$
\begin{gathered}
a=\frac{\sum\left(x_{i}-\bar{x}\right)\left(y_{i}-\bar{y}\right)}{\sum\left(x_{i}-\bar{x}\right)^{2}}, \\
b=\bar{y}-a \bar{x} .
\end{gathered}
$$

\section{Validation and Comparison}

In the first place we are going to recognize our numerical problem with asymptotic solution pursued by Elder [2]. According to his computation if is the velocity of the fluid at the exit of the channel while moving through the screen then we hold a relation for the small-angle carried out by the rule.

$$
\frac{\left(\frac{u}{u_{\text {in }}}-1\right)\left(1+\eta+\kappa \cos ^{2}(\theta)\right)}{(1-\eta) \tan (\theta) \kappa \cos ^{2}(\theta)}=\frac{2}{\pi} \log \left(\cot \left(\frac{\pi y}{2}\right)\right) .
$$

Here, the left-hand side is considered numerical and contrasted with the right-hand side and are together known as stream-wise velocity. We have checked the equation (24) for the Reynolds number. Figure 3 shows the stream-wise velocity worked out for specific Reynolds number with the indices of $0.7,1$ and 1.3, which shows that the technique of Galerkin's Least Square (GLS) scheme is with the satisfactory agreement with the asymptotic solution obtained by Elder [2]. 


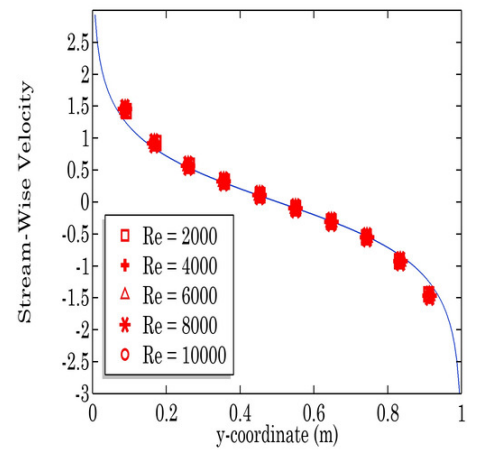

(a) $\mathrm{n}=0.7$

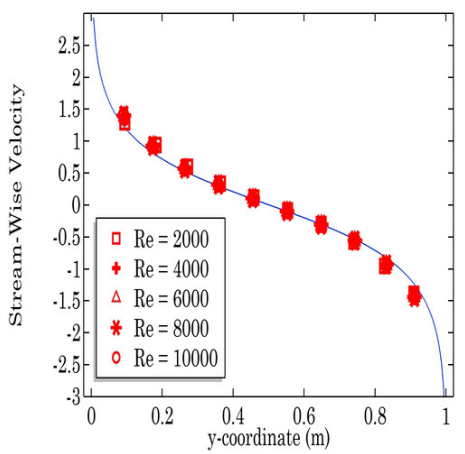

(b) $n=1$

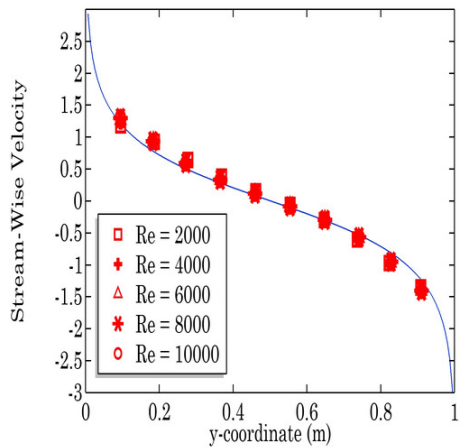

(c) $\mathrm{n}=1.3$

Figure 3: Stream-wise velocity when screen is at $10^{\circ}$ for all Reynolds number from 1000 to 10,000

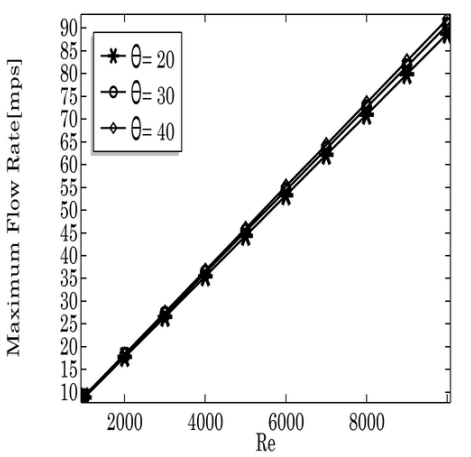

(a) $\mathrm{n}=0.7$

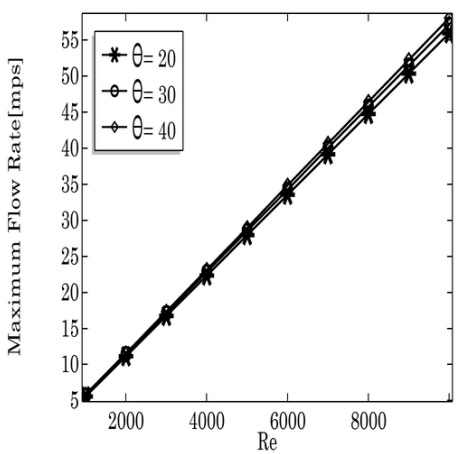

(b) $\mathrm{n}=0.8$

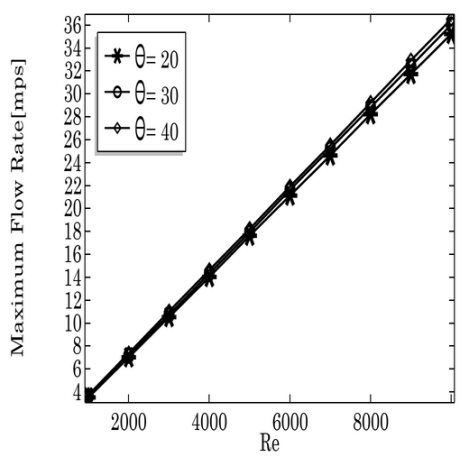

(c) $\mathrm{n}=0.9$

Figure 4: Maximum flow rate against the Reynolds number for different values of $\mathrm{n}$

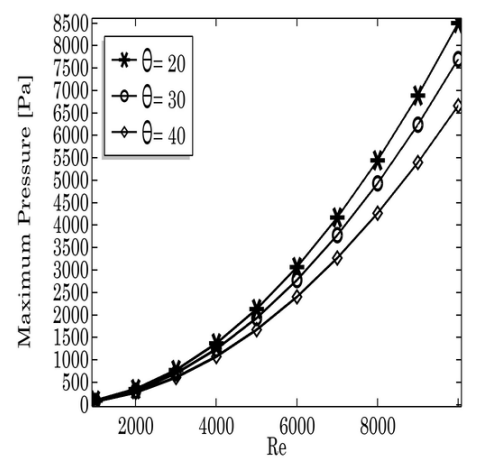

(a) $\mathrm{n}=0.7$

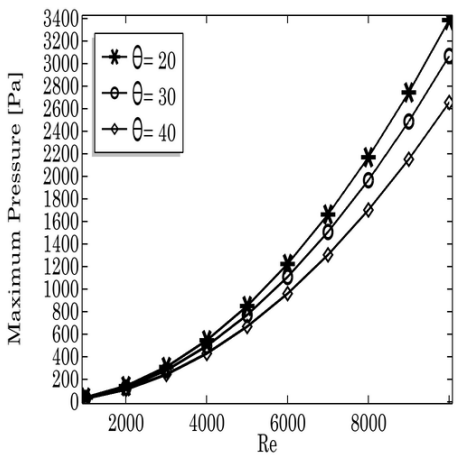

(b) $\mathrm{n}=0.8$

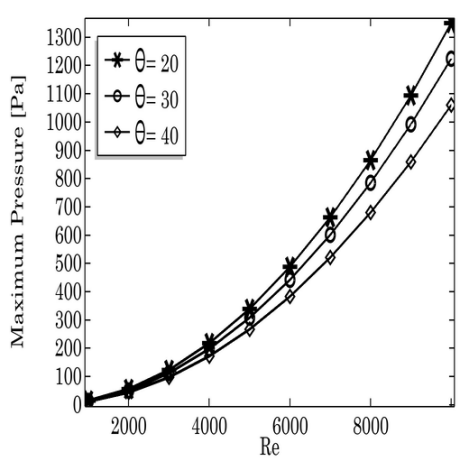

(c) $\mathrm{n}=0.9$

Figure 5: Maximum pressure against the Reynolds number for different values of $n$ 


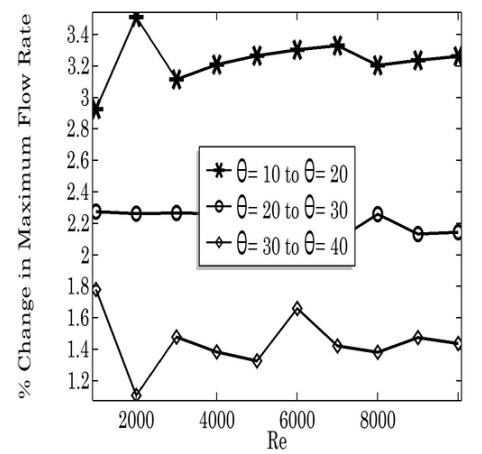

(a) $\mathrm{n}=0.7$

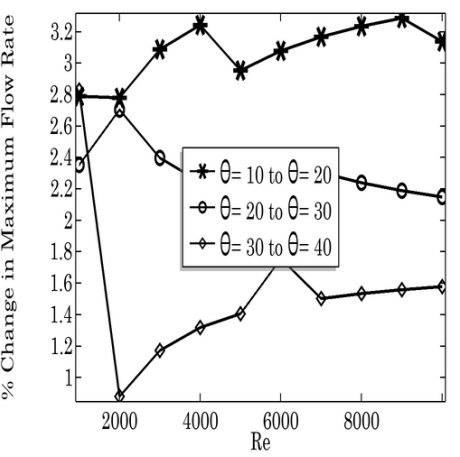

(b) $\mathrm{n}=0.8$

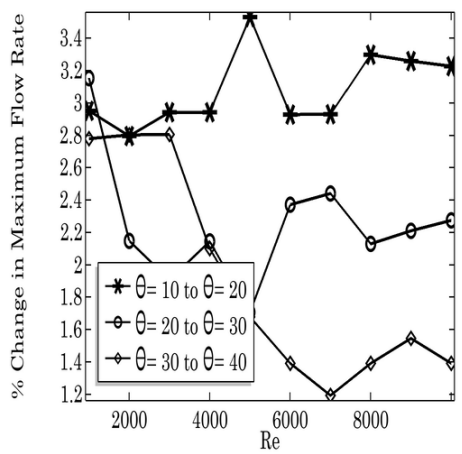

(c) $\mathrm{n}=0.9$

Figure 6: Percent change in maximum flow rate against the Reynolds number for different values of $n$

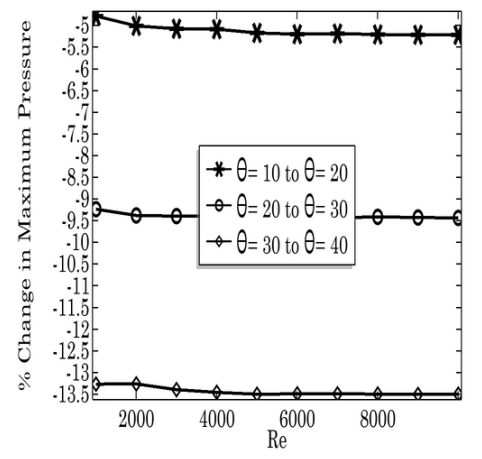

(a) $\mathrm{n}=0.7$

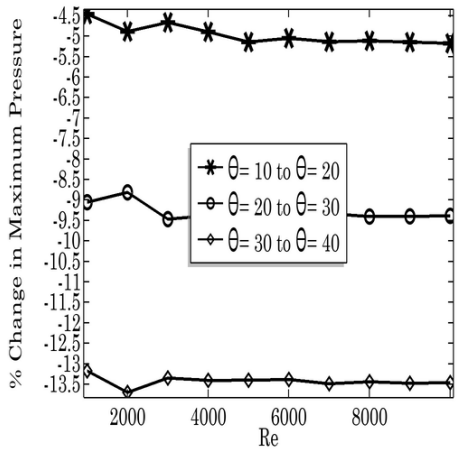

(b) $\mathrm{n}=0.8$

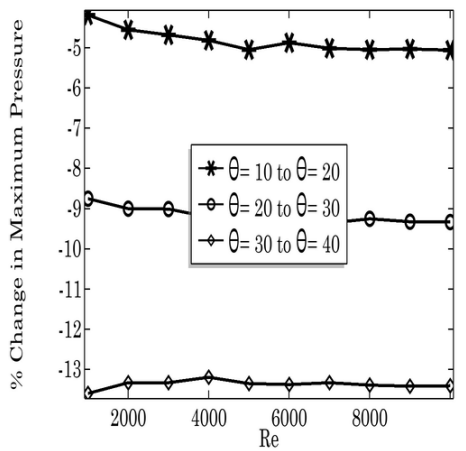

(c) $\mathrm{n}=0.9$

Figure 7: Percent change in maximum pressure against the Reynolds number for different values of $n$

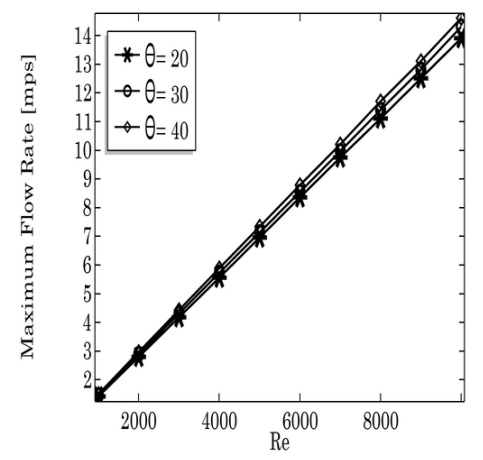

(a) $\mathrm{n}=1.1$

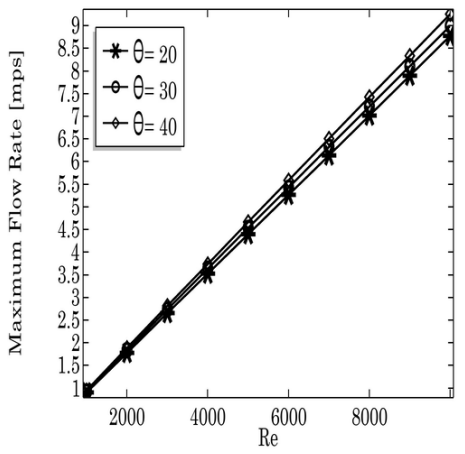

(b) $\mathrm{n}=1.2$

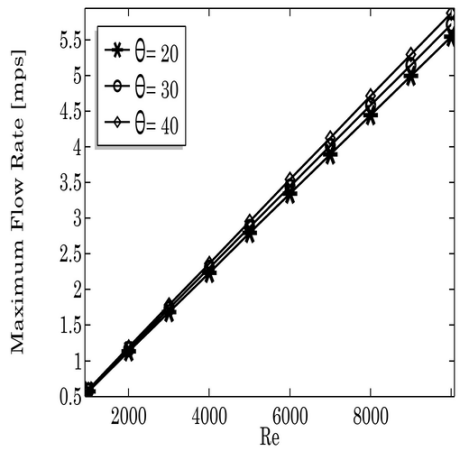

(c) $\mathrm{n}=1.3$

Figure 8: Maximum flow rate against the Reynolds number for different values of $n$ 


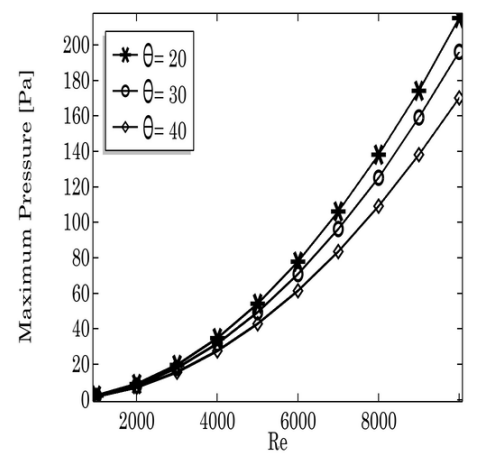

(a) $\mathrm{n}=1.1$

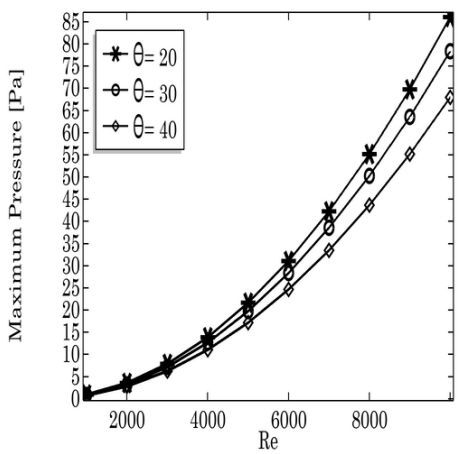

(b) $\mathrm{n}=1.2$

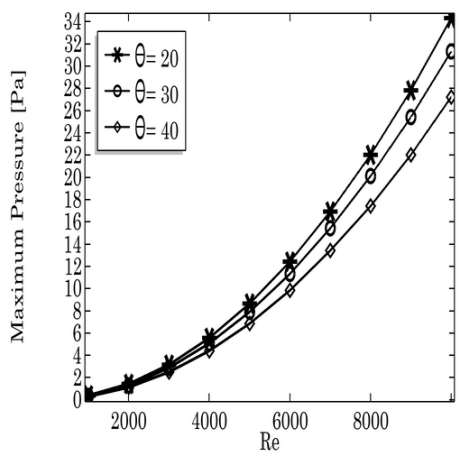

(c) $\mathrm{n}=1.3$

Figure 9: Maximum pressure against the Reynolds number for different values of $n$

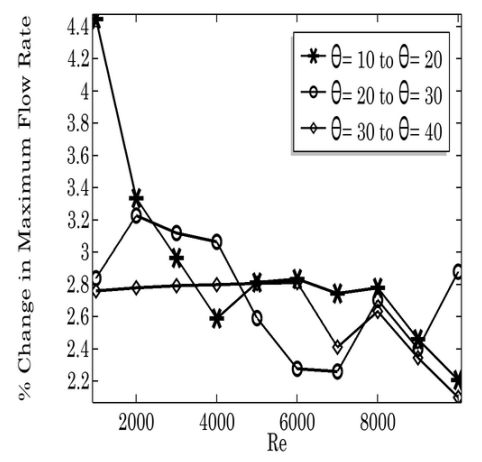

(a) $\mathrm{n}=1.1$

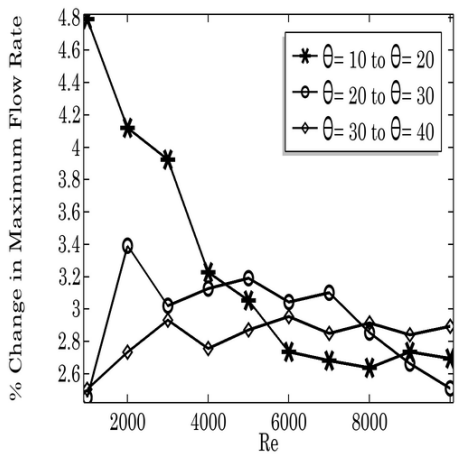

(b) $\mathrm{n}=1.2$

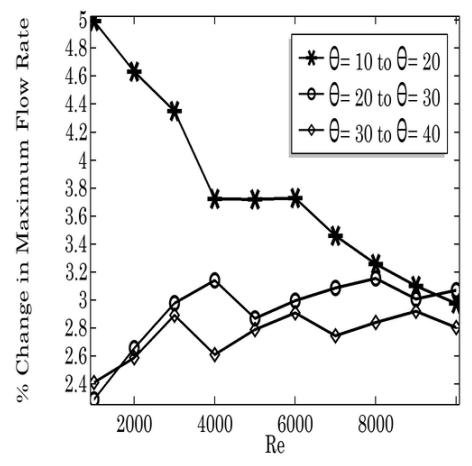

(c) $\mathrm{n}=1.3$

Figure 10: Percentage change in maximum flow rate against the Reynolds number for different values of $n$

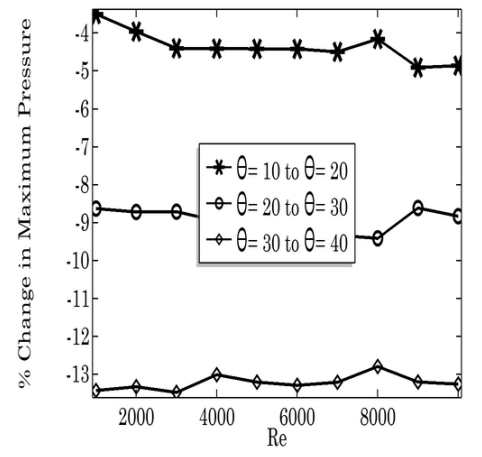

(a) $\mathrm{n}=1.1$

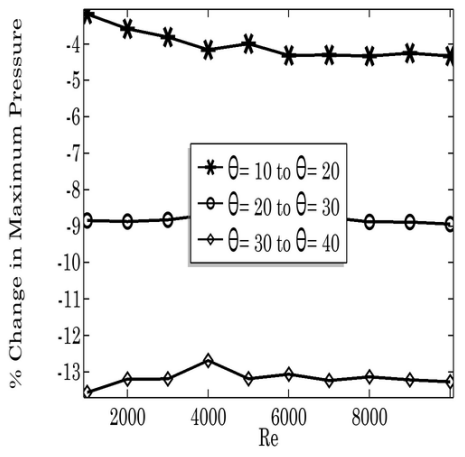

(b) $\mathrm{n}=1.2$

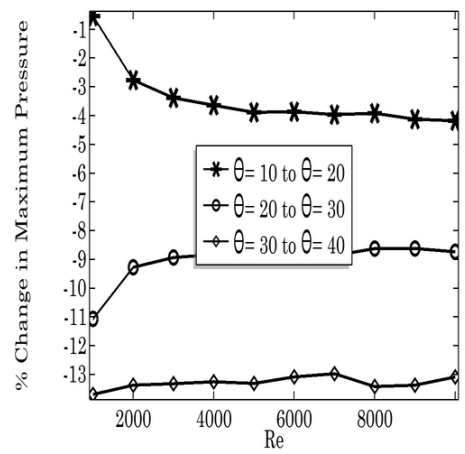

(c) $\mathrm{n}=1.3$

Figure 11: Percentage change in maximum pressure against the Reynolds number for different values of $n$ 


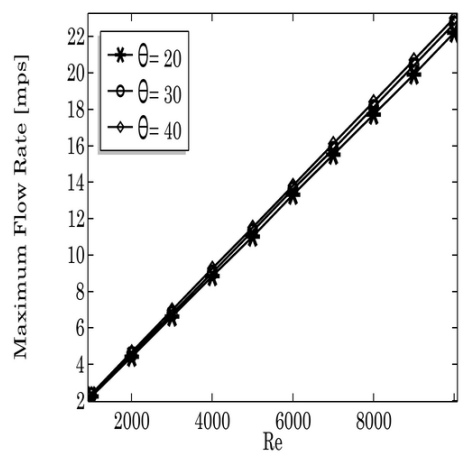

(a) Maximum flow rate

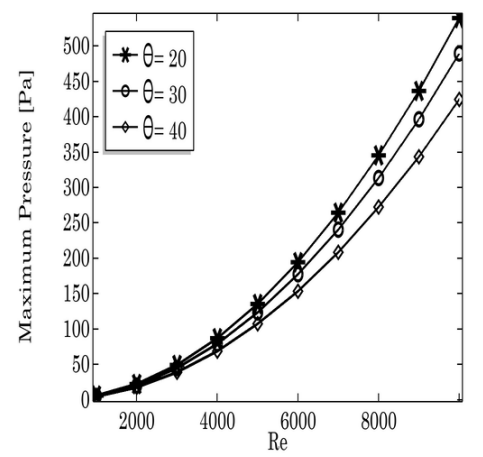

(b) Maximum pressure against

Figure 12: Maximum flow rate and maximum pressure against Reynolds number for $n=1$
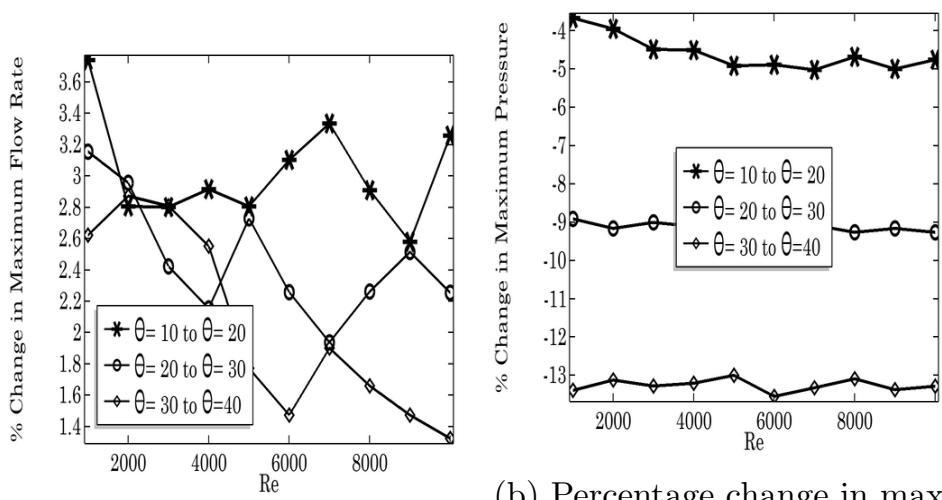

(b) Percentage change in maxi-

(a) Percentage change in maxi- mum pressure against Reynolds mum flow rate number

Figure 13: Percentage change in maximum flow rate and maximum pressure against Reynolds number for $n=1$

\section{Result Discussion}

We divide the discussion into three parts. In the first part is devoted to shear thinning impact i.e. for $(n=0.7,0.8$ and 0.9$)$. In the second part we will discuss about shear thickening impact i.e. $(n=1.1,1.2$ and 1.3). In the end we will have discussion on impacts about Newtonian fluid at $(n=1)$.

\subsection{Shear Thinning fluid}

In the fragment, we are working to designate the fall outs of shear thinning fluid attained by applying the discretization procedure of Least Square Finite Element Method via the COMSOL MULTIPHYSICS. The power law model of generalized Newtonian fluids has been experienced for the indices $n=0.7, n=0.8$ and $n=0.9$. The flow decorations 
Table 2: Regression Analysis for Shear-Thinning fluid.

\begin{tabular}{|c|c|c|c|}
\hline Index & Angle & $\begin{array}{l}\text { Optimum Velocity } \\
\text { Linear Regression } \\
V_{\max }\end{array}$ & $\begin{array}{l}\text { Optimum Pressure Quadratic Regression } \\
P_{\max }\end{array}$ \\
\hline 0.7 & $\begin{array}{l}20 \\
30 \\
40\end{array}$ & $\begin{array}{l}0.00888 R_{e}-0.08667 \\
0.00907 R_{e}-0.06 \\
0.0092 R_{e}-0.07\end{array}$ & $\begin{array}{l}0.4+4 * 10^{-4} R e+8.49 * 10^{-5} R e^{2} \\
0.1+7 * 10^{-4} R e+7.686 * 10^{-5} R e^{2} \\
0.57+6.4 * 10^{-4} R e+6.646 * 10^{-5} R e^{2}\end{array}$ \\
\hline 0.8 & $\begin{array}{l}20 \\
30 \\
40\end{array}$ & $\begin{array}{l}0.0056 R_{e}-0.088 \\
0.0057 R_{e}-0.056 \\
0.0058 R_{e}-0.08533\end{array}$ & $\begin{array}{l}-0.2+6.3 * 10^{-4} R e+3.3781 * 10^{-5} R e^{2} \\
0.06+5.9 * 10^{-4} R e+3.06 * 10^{-5} R e^{2} \\
-0.267+7.57 * 10^{-4} R e+2.645 * 10^{-5} R e^{2}\end{array}$ \\
\hline 0.9 & $\begin{array}{l}20 \\
30 \\
40\end{array}$ & $\begin{array}{l}0.00353 R_{e}-0.06733 \\
0.0036 R_{e}-0.08 \\
0.00365 R_{e}+0.0333\end{array}$ & $\begin{array}{l}-0.387+5.418 * 10^{-4} R e+1.3439 * 10^{-5} R e^{2} \\
-0.17+5.03 * 10^{-4} R e+1.2182 * 10^{-5} R e^{2} \\
-0.36+6.1 * 10^{-4} R e+1.0532 * 10^{-5} R e^{2}\end{array}$ \\
\hline
\end{tabular}

Table 3: Regression Analysis for Shear-Thickening fluid.

\begin{tabular}{|c|c|c|c|}
\hline Index & Angle & $\begin{array}{l}\text { Optimum Velocity } \\
\text { Linear Regression } \\
V_{\max }\end{array}$ & $\begin{array}{l}\text { Optimum Pressure Quadratic Regression } \\
P_{\max }\end{array}$ \\
\hline 1.1 & $\begin{array}{l}20 \\
30 \\
40\end{array}$ & $\begin{array}{l}0.00139 R_{e}+0.01267 \\
0.00142 R_{e}+0.024 \\
0.00145 R_{e}+0.05333\end{array}$ & $\begin{array}{l}-0.24+2.14 * 10^{-4} R e+2.13076 * 10^{-6} R e^{2} \\
0.104+1.1 * 10^{-5} R e+1.974 * 10^{-6} R e^{2} \\
-0.22+6.5 * 10^{-5} R e+1.6948 * 10^{-6} R e^{2}\end{array}$ \\
\hline 1.2 & $\begin{array}{l}20 \\
30 \\
40\end{array}$ & $\begin{array}{l}0.00087 R_{e}+0.02213 \\
0.0009 R_{e}+0.03827 \\
0.0009 R_{e}+0.0368 \\
\end{array}$ & $\begin{array}{l}0.001+2.66 * 10^{-5} R e+8.5741 * 10^{-7} R e^{2} \\
-0.047+5.8 * 10^{-5} R e+7.7787 * 10^{-7} R e^{2} \\
-0.076+7.7 * 10^{-5} R e+6.724 * 10^{-7} R e^{2}\end{array}$ \\
\hline 1.3 & $\begin{array}{l}20 \\
30 \\
40\end{array}$ & $\begin{array}{l}0.0005 R_{e}+0.0232 \\
0.00057 R_{e}+0.01973 \\
0.00059 R_{e}+0.01733\end{array}$ & $\begin{array}{l}-0.0193+3.09 * 10^{-5} R e+3.4014 * 10^{-7} R e^{2} \\
-0.024+2.65 * 10^{-5} R e+3.1078 * 10^{-7} R e^{2} \\
-0.018+2.09 * 10^{-5} R e+2.6989 * 10^{-7} R e^{2}\end{array}$ \\
\hline
\end{tabular}

through the screen for extreme flow rate and extreme pressure are described with the Reynolds numbers. It is obsed that to mark the fluids viscosity lower, the fluids get motivated to increase its speed while flowing through the channel and reducing the pressure.

The structure for the maximum flow rate and maximum pressure is investigated for Re from 1000 to 10, 000. Our purpose to pursue the gradient for maximum flow rate as well pressure in the edges of the domain in overall range of non-dimensional number Re. Figure 4 illustrates maximum flow rate abutting the Reynolds number. The maximum flow rate occurred in the edges of the channel is increased with the Reynolds number linearly subject to the power law indexes $0.7,0.8$ and 0.9 . Figure 5 presents the consequences for maximum pressure at $0.7,0.8$ and 0.9 in the respective Reynolds number for the shear thinning fluid. Escorted by boosting Reynolds number, the maximum pressure at the power law index from $0.7,0.8$ and 0.9 do not recognize a linear order. However, 
Table 4: Regression Analysis for Newtonian fluid.

\begin{tabular}{|l|l|l|l|}
\hline \multirow{3}{*}{ Index } & Angle & $\begin{array}{l}\text { Optimum Velocity } \\
\text { Linear Regression } \\
V_{\max }\end{array}$ & Optimum Pressure Quadratic Regression \\
\hline \multirow{3}{*}{1} & 20 & $0.00222 R_{e}-0.03667$ & $P_{\max }$ \\
& 30 & $0.00227 R_{e}-0.018$ & $-0.25+3.39 * 10^{-5} R e+5.382 * 10^{-6} R e^{2}$ \\
& 40 & $0.00229 R_{e}+0.066$ & $-0.23+3.17 * 10^{-4} R e+4.2083 * 10^{-6} R e^{2}$ \\
& &
\end{tabular}

a parabolic pattern is identified from the figure 4 around the edges of the channel. This can be further identified the gradient of the pressure is increasing intended each power law index. Using the linear regression for the optimum flow rate and quadratic regression for the optimum pressure we can estimate the best fit curves for the both parameters ranging the Reynolds numbers, see Table 2 . It has been urged that by turning the screen in clockwise direction when attached at the middle of the channel, this influence affects to raise the flow rate or speed of the fluid as well as reduces the optimum pressure.

Our goal in the current work is to find the percentage innovation of both parameters when it is turning from 100 to 200, 200 to 300 and 300 to 400. We turn up that this will be too satisfactory to reveal such type of observation with enhancing Reynolds number from 1000 to 10,000 for the shear thinning fluids with indices $0.7,0.8$ and 0.9. Figure 6 sets out that the change in flow rate for $0.7,0.8$ and 0.9 from 200 to 300 do not show the oscillation away from 12 percentage change in the flow rate. Whereas the percentage change in flow rate while turning up the screen from 300 to 400 degrees for the indices 0.7, 0.8 and 0.9 shows more flexibility and change up to the maximum of 20 percentages. The percent change in flow rate for the shear thinning fluid at 0.7 shows more firmness i.e. it does not go away from the center of the fluctuations and while the flow rate for the indices 0.9 shows more fluctuation. The outcomes in percentage change in the optimum pressure while the screens are turning in clockwise direction are conferred in figure 7 , shows that increment in pressure shows stability with that of Reynolds number. It shows that for all the indices while screens are turning from 100 to 200, 200 to 300 and 300 to 400 degrees, the increment in the pressure impacts about the same percentage.

\subsection{Shear thickening fluid}

The scrutiny has been prolonged to notice the conduct of shear thickening fluid for the air flow by norms of the solution attained by solving in-compressible Navier Stokes equation by launching the Galerkins strategy of least square. Appropriate to the determination, we are practical the power law indexes 1.1, 1.2 and 1.3 in the power law representation of the non-Newtonian. The dilemma has been elucidated well by using the commercial software Comsol Multiphysics. Supposed to the shear thinning fluid, shear thickening fluid is also studied for extreme fluid rate and extreme pressure exerted in the 
channel along with the increment of the flow rate and pressure while screens are turning up in clockwise direction away from the center of the channel from one degree to another. The consequences for extreme flow rate and pressure around the edges of the domain while air is coursing through the screen are offered through the figure 8 and 9. Certainly, the extreme flow rate holds the linear relationship with that of the Reynolds number and while the pressure is consistently shown the parabolic pattern with that of the Reynolds number. Referring to figure 8 and 9 we can judge the equations for extreme flow rate and maximum pressure around the domain by using the linear regression and quadratic regression methods described by equation 17 and equation 21 respectively, see table 3 . Further, we can perceive by turning the screen in clockwise course, the extreme flow rate is little bit boosting or improving. For example, the flow rate at 300 is more than 200 degrees for indices 1.1, 1.2 and 1.3.

In the case, where we have inspected the increment in flow rate as well as pressure while the screen is turning in clockwise direction, see figure 10 and figure 11 . The percent increment is dropping unceasingly while the screen is turning from 100 to 200 degrees with boosting the Reynolds number, whereas for 200 to 300 and 300 to 400 degrees is increasing. The percentage increment for all indexes with the boosting of Reynolds number reaches up to some equilibrium points. For instance, see figure 10(c), the percent increment for all the turning up screen reaches between 2.8 to 3.2. We expected there would be a certain Reynolds number occurred where this increment stopped. On the other hand, when we look at the percent increment in the pressure for the turning effect of the screen, see figure 11. The percentage increment in the pressure while the screen is turning up from 100 to 200 and 200 to 300 degrees does not show the significant increment with the boosting Reynolds number. Nevertheless, we can comment or can give the significance in the percentage change in the maximum pressure while considering the turning effect from 300 to 400 .

\subsection{Newtonian Fluids}

The power-law model of the Newtonian is laboring with the index $n=1$ to scrutinize the fluid flow performance through the screen attached at the particular angles from 100 to 450 degrees in the clockwise direction. For the determination, the in-compressible Navier Stokes equation has been discretized consuming the least square Galerkins scheme and the entire impacts are confirmed with the enhancement of non-dimensional Reynolds number. The purpose of testing the Newtonian fluid through the screens is to plaid the difference in the behavior of optimized flow rate and pressure while the fluid elegant through the screen between the parallel plates. The optimum flow rate is increasing linearly with growing Reynolds number from 1000 to 10,000 and also by refining or turning the screen from 20 to 30 or 30 to 40, the optimum flow rate is boosting little bit, see figure 12(a). Through the optimized pressure and likewise to the shear-thinning fluid and thickening fluid, a parabolic relationship has been established, see figure 12(b). By spotting this we can custom the linear regression as well as parabolic regression procedure we can estimate 
the best fit lines described by table 4 . Which shows the gradient of flow rate is maximum when the screen attached at 400 .

Further, we also calculate the increment the flow rate and pressure while the screens are turning in the unit degree. The increments in percentage are conferred by figure 13(a) and figure 13(b) for the optimized flow rate and pressure respectively with the improvement of the Reynolds number. It outlines from the figure that the optimized flow rate shows the oscillatory behavior with that of the Reynolds number and the Reynolds number shows some firmness with that of the pressure while the screen is turning from 100 to 200, 200 to 300 and 300 to 400 degrees.

\section{Conclusion}

The finite element reproduction has been done for the Newtonian and non-Newtonian fluid moved through the parallel plates attached accompanied by the block facing screen boundary conditions. The non-Newtonian power-law model has been taken on for shearthinning $(\mathrm{n}=0.7,0.8$ and 0.9), shear-thickening $(\mathrm{n}=1.1,1.2$ and 1.3) and Newtonian $(\mathrm{n}=1)$ fluid with the usage of the Reynolds number varying from 1000 to 10,000. The entire procedure has been gone through by the commercial software Comsol Multiphysics which utilized the finite element technique of least squares. The maximum flow rate, as well as the pressure, was dealt with along with the percentage increment was looked at while the screens are turning clockwise from one degree to another. It was found that the maximum flow rate is increasing linearly and maximum pressure is increasing in the parabolic association with that of the enhancement of the Reynolds number. Thus, by applying the linear and quadratic regressions course, the regression curves are reckoned and rooted in the tables. The increment in the maximum flow rate and pressure has been debated in the terms of Reynolds number. It was uncovered that for both shear-thinning and Newtonian fluid the increment alternates noticeably with the enhancement of the Reynolds number and at the same time as for the shear thickening fluid with the accretive Reynolds, the increments will consolidate at the equilibrium point. A point will most likely arise where the increment will continue constant or stable no matter how much the Reynolds number is increasing.

\section{References}

[1] Ted Chu Channing R. C. Santiago and K. H. Wang. Study of the head loss associated with a fluid flowing through a porous screen. Research report, Department of Civil and Environmental Engineering, University of Houston,Houston, Texas., 2007.

[2] J. W. Elder. Steady Flow through non-Uniform Gauzes of Arbitrary Shape. Journal of Fluid Mechanics, 5(3):355-368, 1959.

[3] Alexander Fischer and Jens Gerstmann. Flow resistance of metallic screens in liquid, 
gaseous and cryogenic flow. In Dipl.-Ing. Christoph Kirchberger, editor, Fifth European Conference for Aero-Space Science, pages 1-5, Germany, 2013. EUCASS Associates.

[4] V. M. Mohan G.A. Sawant and S. A. Sawant. Study and Analysis of Deck Inclination Angle on Efficiency of Vibration Screen. International Journal of Engineering Development and Research, 4(1):631-635, 2016.

[5] G. Hauke and T. J. R. Hughes. A Unified Approach to Compressible and Incompressible Flows. Computer Methods in Applied Mechanics and Engineering, 113(3-4):389395, 1994.

[6] H. Shaikh M. Abid and M. Asif. Finite elements analysis of fluid flow through the rectangular channel with inclined screens settled at angles. In Second International Conference on Computing, Mathematics and Engineering Technologies, Germany, 2019. EUCASS Associates.

[7] M. Teitel. Using Computational Fluid Dynamics Simulations to Determine Pressure Drops on Woven Screens. Biosystems Engineering, 105(2):172-179, 2010. 